\title{
Shorten Myopic Axial Length with Repeated Low-lever Laser Light Therapy
}

\author{
Zhou Lei ${ }^{1}$, Qiu Kaikai ${ }^{2, *}$, William Bruce ${ }^{3}$ \\ ${ }^{1}$ Ning Bo Eye Hospital, Ningbo, China \\ ${ }^{2}$ Fuzhou South East Eye Hospital, Fuzhou, China \\ ${ }^{3}$ Seattle Vision Care Center, Seattle, USA
}

Email address:

qkk1229@qq.com (Qiu Kaikai)

\section{To cite this article:}

Zhou Lei, Qiu Kaikai, William Bruce. Shorten Myopic Axial Length with Repeated Low-Lever Laser Light Therapy. International Journal of Ophthalmology \& Visual Science. Vol. 6, No. 2, 2021, pp. 144-149. doi: 10.11648/j.ijovs.20210602.22

Received: May 1, 2021; Accepted: June 18, 2021; Published: June 26, 2021

\begin{abstract}
Purpose: This study was to confirm that repeated low-lever laser light therapy (LLLT) could control myopic axial length (AL) elongation. Methods: Twenty myopia, 5 to 13 years old, were included in this study. All subjects kept daily repeated therapy with LLLT for mean 3 months; According to the baseline AL ( $>24.40 \mathrm{~mm}$ or not), and ages ( $>10$ years old or not), we built two age groups and two AL groups. The AL between baseline and follow-up as well as different groups were all tested by SPSS 26.0, respectively. Results: $85 \%$ and $75 \%$ of AL were shortened for the right and left compared with those of baseline, respectively. The follow-up right and left $\mathrm{AL}$ were both significantly shortened $(\mathrm{P}=.002, \mathrm{P}=.003$, respectively) from baseline mean right AL $24.52 \pm 1.01 \mathrm{~mm}$, left AL $24.51 \pm 0.96 \mathrm{~mm}$, to $24.41 \pm 0.98 \mathrm{~mm}(-0.11 \mathrm{~mm}$ right AL shortened amount) and 24.42 \pm 0.93 ( $-0.09 \mathrm{~mm}$ left AL shortened amount), respectively. Long baseline AL group was significantly $(\mathrm{P}=.02, \mathrm{P}=.03$ ) shortened more AL than those of small baseline AL group for both right and left $\mathrm{AL}(-0.17 \mathrm{~mm}$ versus $-0.05 \mathrm{~mm}, \mathrm{P}<.001$, respectively). From age group aspect, although it was also significant difference changed (shortened) for right $\mathrm{AL}(\mathrm{P}=.008)$, but not for left $\mathrm{AL}$ $(\mathrm{P}=.051)$. Conclusions: LLLT could control myopia axial length progression.
\end{abstract}

Keywords: Myopia, Low-Lever Laser Light, Axial Length

\section{Introduction}

Most previous evidence-based control methods for myopia progression control was only to reduce axial length elongation rates, such as atropine, [1] orthokeratology, [2] Misight, [3] or even the combined therapy of atropine with orthokeratology resulting in approximately $0.09 \mathrm{~mm} /$ year axial elongation. [4] And it was a popular prejudice that axial length cannot be shortened for the long term spanning months or years. Although, with modern advanced optical coherent measurement, we can detect very small amounts of change, as little as 0.01-0.02 mm axial length change, either elongation or reduction, for short term - one night change, such as circadian rhythms. [5-6]

To the best of our knowledge, there were few reports of axial length shortening in human clinical or animal studies.

And there was a legend in China that the axial length of a highly myopic, amblyopic child was found to have a $0.30 \mathrm{~mm}$ axial length reduction after one year of therapy with a red light instrument. A low-lever laser diode medical device for the treatment of amblyopia. The result was later presented in a case report that was rejected directly because the finding of axial length reduction for high myopia was inconsistent with the traditional concept that axial length could never be shortened for a longer term and for such an amount $(0.30 \mathrm{~mm})$.

However, as it was reconfirmed in other myopia cases; A group of scientists initiated a randomized clinical trial registered at clinicaltrials. gov (NCT04073238, n=264) named as "Efficacy of Repeated Low-Level Red-Light Therapy in Myopia Control" confirmed that the AL reduction was statistically significant at $P<.001$ both for one month and at the end of six month. But they have not yet published their results in papers; Their method and promising result had already known to many Chinese forums such as CCOS and online videos, which made such kinds of medical device very popular in mainland during COVID-19 epdemic period and nowadyas.

One recent paper with randomized clinical trial design, published in 2021, from affiliated Eye Hospital of Nanchang 
University of China, they compared three groups (spectacles vs. Orhtokeratology vs. LLLT with final number of each group: 74 vs. 81 vs. 74) of myopia progression amount for 6 months. And they confirmed that slightly better myopia control was observed with LLLT treatment than with overnight OK lens-wearing with changes in axial length (AL) $0.23 \pm 0.06$ $\mathrm{mm}$ for wearing single-vision distance spectacles for 6 month, $0.06 \pm 0.15 \mathrm{~mm}$ for children wearing OK lens and $-0.06 \pm 0.15$ $\mathrm{mm}$ for children treated with LLLT for 6 month.[7]

In animal studies, the light experiment results were contradictory, depending on the animal species;[8] Chicks,[9-10] rainbow trout (Oncorhynchus mykiss)[11] and guinea pigs (Cavia porcellus) [12] became more myopic with 12 hours of red light therapy while refractive development in infant rhesus monkeys [13] or juvenile and adolescent tree shrews [14-15] ended in more hyperopia.

This study was to confirm whether, in human children, axial length would be shortened with red light therapy for the purpose of myopia control.

\section{Methods}

The study was conducted in accordance with of the Helsinki Declaration, which was approved by the local ethics committee. Subjects were included from December 2019 to November 2020 with follow-up at least one month red light treatment. The red light therapy instrument was a semiconductor laser diode with wavelength $635 \mathrm{~nm} \pm 10 \mathrm{~nm}$, and mean $0.38 \mathrm{~mW}$ energy to either eye for the same treatment. All the myopic subjects requested red light therapy due to an increase in myopia progression with $\mathrm{AL}$ elongation. Before therapy, all 20 myopic subjects underwent routine examinations in the hospital including anterior and posterior segments evaluation to exclude other diseases. Written informed consent to this study was obtained from the supervisors or guardians of the myopic children following the prescription below: 3 minutes per session, two sessions per day with at least a 4 hour interval between the two sessions of a continuous, bilateral red light therapy,

The device is a desktop, single wave, red light amblyopia therapy instrument (Figure 1. Jilin Longda Medical Devices Co., Ltd. China); A Class II medical device and widely used in many Chinese hospitals since 2006. And the target location was the macula of the retina. According to ICE laser classification and U.S. FDA laser classification, its radiation category belonged to Class I. According to the China national laser product safety standard (GB7247. 1-2012), radiation Class I could be used safely into the eyes.

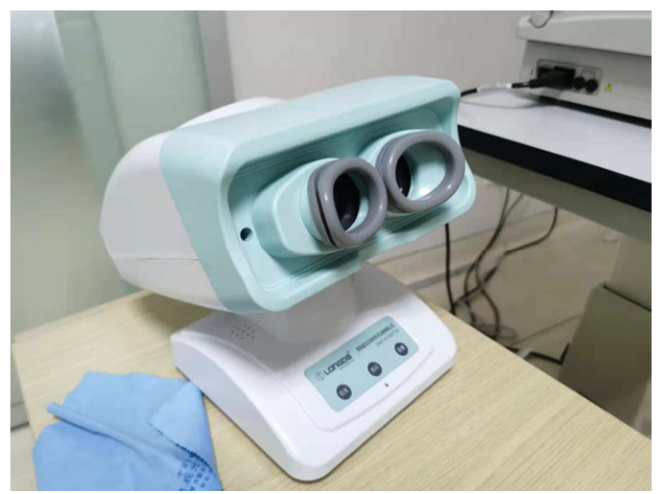

Figure 1. Red Light Therapy Instrument in Ning Bo Eye Hospital.

AL data was recorded for each subject with an IOLmaster 500 (Carl Zeiss Meditec AG, Jena, Germany). Best corrected visual acuity was recorded at baseline and at follow-up.

Clinical follow-up interval was 1 3 months. The results for axial length, vision, refraction and some OCT measurements were all examined during follow-ups. These results were taken to monitor any potential side effects of the treatment. None were found. Only axial length measurments were collected and statistically analyzed.

Axial Length was described by mean \pm standard error. Only the latest follow-up axial length and the baseline axial length were analyzed by the paired $t$-test for either eye, respectively.

Statistically significant difference was defined as $P<.05$. And all statistical analyses were performed with software of IBM SPSS Statistics 26.0 (SPSS Inc., Chicago, Illinois, U.S.A).

\section{Results}

A comparison of baseline axial length and latest follow-up axial length of the twenty myopic subjects (13 males and 11 females), is illustrated in Table 1. The mean axial lengths were significantly shortened, from the baseline $24.38 \pm 1.19$ $\mathrm{mm}$ and $24.33 \pm 0.99 \mathrm{~mm}$ to the latest follow-up $24.27 \pm 1.14$ $\mathrm{mm}$ and $24.21 \pm 0.99 \mathrm{~mm}$, for the right and left eyes respectively $(P<.001, P=.001$; paired $t$ test $)$.

Table 1. Paired t test of Comparing Baseline Axial Length with Follow-up Axial Length.

\begin{tabular}{llllll}
\hline & & Mean & Number & SD & P value \\
\hline \multirow{2}{*}{ Paired 1 } & Baseline Right AL $(\mathrm{mm})$ & 24.516 & 20 & 1.013 & .002 \\
& Follow-up Right AL (mm) & 24.409 & 20 & 0.977 & 0.957 \\
\multirow{2}{*}{ Paired 2 } & Baseline Left AL (mm) & 24.509 & 20 & 0.930 & .003 \\
\hline
\end{tabular}

Footnote: SD meant Standard Deviation.

At baseline, subjects were 3 to 13 (Mean \pm SD 8.0 \pm 2.6 ) years old. The Spherical equivalent was $-0.75 \sim-5.00$ (Mean $\pm \mathrm{SD},-2.59 \pm 1.96) \mathrm{D}$, and -0.75 to -4.50 (Mean \pm SD $-2.47 \pm$ $1.85) \mathrm{D}$ for the right and left eyes, respectively. And the mean therapy month was $3.3 \pm 1.4$ months of all. Each eye had no visual acuity changes during the follow-up therapy. No one complained of reduced vision or any comfort issues during the follow-up consultations.

The average monthly axial length reduction was $0.031 \pm$ $0.04 \mathrm{~mm}$, and $0.025 \pm 0.04 \mathrm{~mm}$ for the right and left eyes, 
respectively; At the same time, the monthly mean reduction in refractive power was $0.06 \mathrm{D}$ for either eye group. There was no significant binocular axial length difference change from the follow-up axial length to the baseline axial length $(P=.240$, paired $t$-test $)$.

Considering the two different age classifications, illustrated in table 2, Group 2 with mean age of $11.3 \pm 1.4$ years old, had significantly shorter baseline axial length $(P=.008)$ and shorter follow-up axial length $(P=.017)$, but only limited to the right eye, compared to that of Group 1 with mean age of $7.2 \pm 0.9$ years old. There was no statistical significance in the left eyes $(P=.051$ for the baseline and $P=.067$ for the follow-up).

Table 2. Axial Length Comparing between young and old Age Groups (Group $1<10$ years old, Group $2>10$ years old).

\begin{tabular}{|c|c|c|c|c|c|}
\hline & Age Group & Number & Mean & SD & P value (t-test) \\
\hline \multirow{2}{*}{ Age (years old) } & 1.00 & 13 & 7.154 & 0.899 & \multirow{2}{*}{$<.001$} \\
\hline & 2.00 & 7 & 11.286 & 1.380 & \\
\hline \multirow{2}{*}{ Baseline Right AL (mm) } & 1.00 & 13 & 24.097 & .712 & \multirow{2}{*}{.008} \\
\hline & 2.00 & 7 & 25.293 & 1.074 & \\
\hline \multirow{2}{*}{ Follow-up Right AL (mm) } & 1.00 & 13 & 24.039 & 0.688 & \multirow{2}{*}{.017} \\
\hline & 2.00 & 7 & 25.094 & 1.110 & \\
\hline \multirow{2}{*}{ Left AL at Baseline (mm) } & 1.00 & 13 & 24.207 & 0.900 & \multirow{2}{*}{.051} \\
\hline & 2.00 & 7 & 25.070 & 0.844 & \\
\hline \multirow{2}{*}{ Left AL at Follow-up (mm) } & 1.00 & 13 & 24.142 & 0.851 & \multirow{2}{*}{.067} \\
\hline & 2.00 & 7 & 24.937 & 0.901 & \\
\hline \multirow{2}{*}{ Binocular AL Difference at Baseline (mm) } & 1.00 & 13 & -0.11 & 0.52 & \multirow{2}{*}{.147} \\
\hline & 2.00 & 7 & .22 & .35 & \\
\hline \multirow{2}{*}{ Binocular AL Difference at Follow-up (mm) } & 1.00 & 13 & -.103 & .460 & \multirow{2}{*}{.184} \\
\hline & 2.00 & 7 & .157 & .328 & \\
\hline \multirow{2}{*}{ Average Months of Therapy } & 1.00 & 13 & 2.692 & .7511 & \multirow[b]{2}{*}{.583} \\
\hline & 2.00 & 7 & 4.571 & 1.718 & \\
\hline
\end{tabular}

Footnotes: SD was meant Standard Deviation; AL was meant Axial Length.

There were no child with age of 10 year old at baseline.

Table 3. Long and short AL groups comparing (Group 1: Baseline Right AL $<24.40$ mm; Group 2: Baseline Right AL $>24.40$ mm).

\begin{tabular}{|c|c|c|c|c|c|}
\hline & R AL group & Number & Mean & SD & P value \\
\hline \multirow{2}{*}{ Age (years old) } & 1 & 11 & 7.27 & 1.27 & .000 \\
\hline & 2 & 9 & 10.22 & 2.22 & \\
\hline \multirow{2}{*}{ Right Baseline AL (mm) } & 1 & 11 & 23.799 & .401 & .000 \\
\hline & 2 & 9 & 25.391 & .819 & \\
\hline \multirow{2}{*}{ Right Follow-up AL (mm) } & 1 & 11 & 23.748 & .409 & .000 \\
\hline & 2 & 9 & 25.216 & .852 & \\
\hline \multirow{2}{*}{ Left baseline AL (mm) } & 1 & 11 & 23.936 & .815 & .000 \\
\hline & 2 & 9 & 25.209 & .584 & \\
\hline \multirow{2}{*}{ Left AL follow-up (mm) } & 1 & 11 & 23.877 & .767 & .000 \\
\hline & 2 & 9 & 25.084 & .647 & \\
\hline \multirow{2}{*}{ Baseline Right and Left AL Difference (mm) } & 1 & 11 & -.137 & .563 & .147 \\
\hline & 2 & 9 & .182 & .314 & \\
\hline \multirow{2}{*}{ Follow-up Right and Left AL difference (mm) } & 1 & 11 & -.129 & .497 & \multirow{2}{*}{.184} \\
\hline & 2 & 9 & .131 & .293 & \\
\hline \multirow{2}{*}{ Months } & 1 & 11 & 3.182 & 1.4709 & \multirow{2}{*}{.583} \\
\hline & 2 & 9 & 3.556 & 1.5092 & \\
\hline
\end{tabular}

Footnotes: SD was meant Standard Deviation; AL was meant Axial Length; $\mathrm{R}$ was meant Right Eye; $\mathrm{L}$ was meant Left eye. There was no $\mathrm{AL}=24.40 \mathrm{~mm}$ at baseline in this study.

However, there was a significant axial length shortening phenomena found in the classification of long axial length group and short axial length group $(P<.001)$ at baseline and follow-up $(P<.001)$, for either the right or left $(P<.001)$, respectively. It was estimated at $0.2 \mathrm{~mm}$ shorter from the baseline $25.29 \mathrm{~mm}$ group (Group 2, $\mathrm{n}=7$ ); While at the same time only approximately $0.06 \mathrm{~mm}$ shorter from baseline $24.09 \mathrm{~mm}$ group (Group 1, $\mathrm{n}=13$ ).

\section{Discussion}

These results support binocular red light therapy, over time, could reduce over 70\% myopic axial length elongation which confirms it as an extra superior method to control myopia, especially for high myopia with long baseline axial length as well as those right eye from the older age of myopia children. Also some subjects had been found $0.50 \mathrm{D}$ reduction in 
myopic refraction, compared to that of the baseline. It could be due to the accuracy of optical measurement instrument such as IOLmaster since few auto-refractors or phoropters would have the accuracy of $0.06 \mathrm{D}$ for monthly refraction change.

In addition, according to our data and classification, the longer baseline axial length and the older age would benefit more from this kind of low-lever laser light therapy for myopia. This result with the axial length shortening phenomena would be especially meaningful to those with high myopia, and consequentially, a higher tendency of developing myopia related ocular complications. There were 9 subjects that had a history of orthokeratology that failed to control axial elongation before the low-lever laser light therapy. After starting several months of low-lever laser therapy all of the subjects showed a reduction in the rate of axial elongation and also showed a small reduction in overall axial length. From such examples, it could be concluded that low-lever laser light therapy had more powerful efficacy to control axial length than that from orthokeratology lens. And it was also indicated combining red light therapy with orthokeratology lens could be a strategy for myopia control.

An explanation to the above results--how could low-lever laser light therapy shorten the axial length- could be through the pathway of cytochrome oxidase $\mathrm{C}$. The cDNA microarray technique of gene expression profiles revealed that 111 genes were regulated by red light and most of these genes enhanced cell proliferation or suppressed apoptosis. As well as several genes related to antioxidation and mitochondria energy metabolism also played a role. [16-19]

Although this red light belonged to "low level laser therapy", also known as Photobiomodulation, For myopia, it could be regarded as an innovative method. For neurological and psychological conditions, [19] stroke, [20] pain relief, [21] fibromyalgia [22] as well as age-related macular degeneration, [23] amblyopia, retinopathy of prematurity, diabetic retinopathy, and Leber's hereditary optic neuropathy, it had already gained the attention of the clinical community as a promising approach.[24] And the reason for shortening axial length of myopia could be caused directly by increasing choroidal thickness, choroidal metabolic rate and circulation to avoid scleral hypoxia. High-resolution optical biometrics (Lenstar) showed that the changes in the depth of the vitreous chamber were caused by the increase in the thickness of the choroid and the thickness of the lens for animals exposed to low-lever laser red light.

The three month red light therapy result of a randomized clinical trial $(n=264)$ registered in clinicaltrials. gov (NCT04073238) had significant choroidal thickening for the red light group. In the first month, the mean AL from experimental group got a small but statistically significant shortening of $\mathrm{AL}(P<.001)$ with a lower myopic refraction $(P<.001)$ and thicker choroidal membrane $(P<.001)$ than those of the control group. For the first month, it showed approximately $0.05 \mathrm{~mm}$ shortening of mean AL than that of the baseline. However, AL for the third month was only shortened by a mean of $0.01 \mathrm{~mm}$ than that of baseline AL for the 132 subjects in experimental group.

Animal experiments have found that very young rhesus monkeys developed more hyperopia under dark background conditions than those under white background for some specific monochromatic red light. Additionally, binocular red light therapy developed more hyperopia than monocular therapy. ${ }^{13}$ However, it would be opposite to refractive development for different species under red light; The difference may be caused by different experimental factors including species, experimental duration, circadian rhythm, light intensity, time sensitivity and hormone release, etc. ${ }^{26}$

From Figure 2, it compared two IOLmaster data for a 11-year-old myopia child: At the 3rd month follow-up, the AL shortened $0.05 \mathrm{~mm}$ and $0.06 \mathrm{~mm}$ for the right AL and left AL, respectively, while corneal powers had a small increase. This is from the point of view of corneal curvatures recorded at the same time, the low-lever red laser light therapy had little effect on the corneal power for the purpose of myopia control.

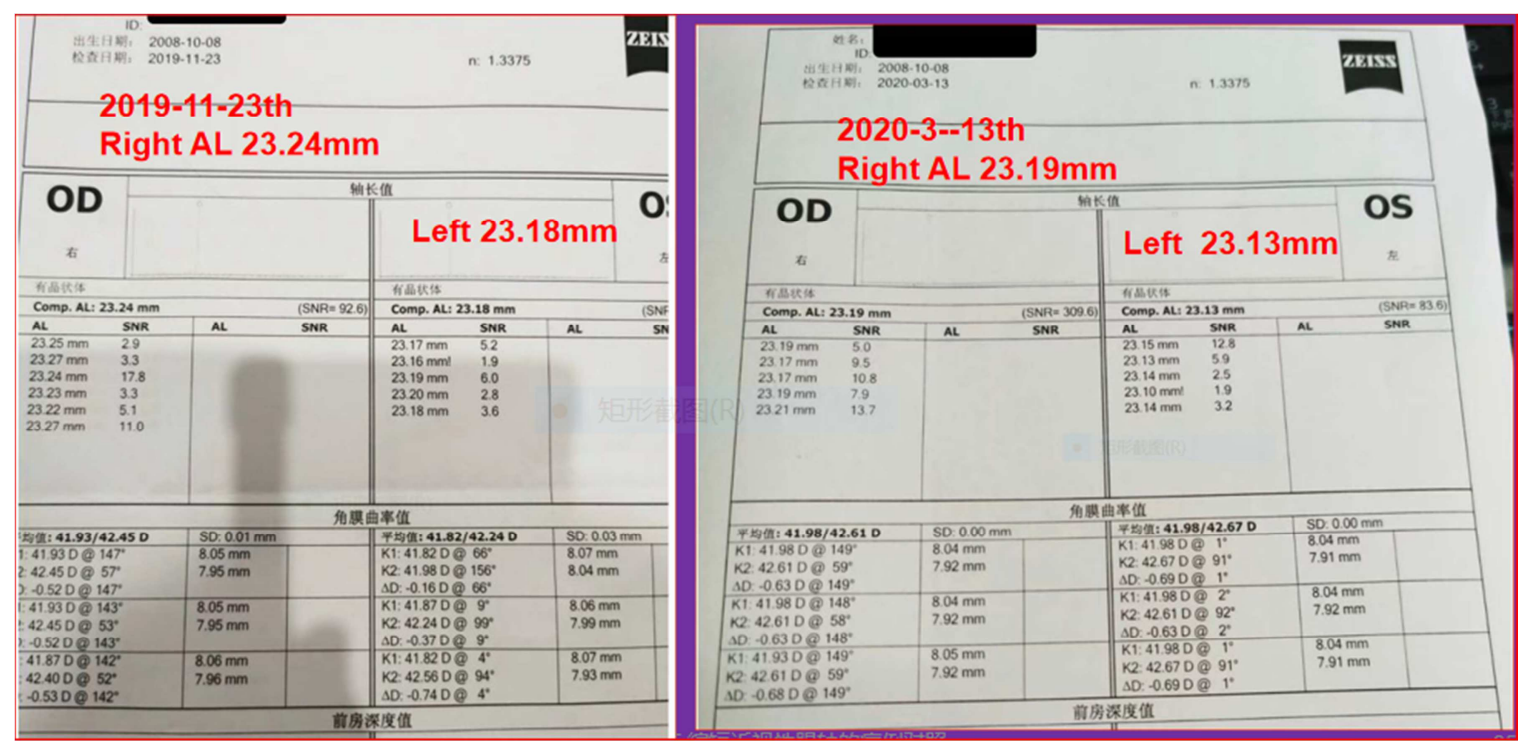

Figure 2. Two axial length measurements comparing. A Myopic Child, Born in 2008, with axial length measured on 2019-11-23th, the right axial length was $23.24 \mathrm{~mm}$ while the left axial length was $23.18 \mathrm{~mm}$. 
Circadian rhythms also affected diurnal changes in refractive power, $\mathrm{AL}$ and choroidal thickness. It was reported as the difference between myopia and emmetropia for 24 hours. [5, 6] With regards to the circadian rhythm effect, it was suggested to implement the red light therapy at night for myopic eyes when the choroid was thicker and the myopic $\mathrm{AL}$ was relatively shorter.

The limitation of this study was a small number participants with neither cycloplegic refraction nor choroidal thickness data to compare to a control group. As well as recording vision but without using statistical analysis. Only a few optical coherent tomographies were examined but without any special analysis. As well as without subjective feedback questionnaires to expose any vision problems.

It is important to note that much more data is needed from future studies on treatment time, treatment frequency, side effects or combining treatment to fully understand the clinical significance of low-lever red laser light therapy. For instance, what manifestation of myopia is the best eligible candidate for red light therapy? Can red light therapy prevent the initial occurrence myopia? Would there be a rebound effect after stopping treatment? There are other therapy pattern issues such as light wavelength, light source and therapy frequency which also require further study to find the optimal treatment model.

\section{Conclusions}

Low-lever red laser laser light therapy could become a very promising and powerful medical method to control myopia progression with a relatively small amount of axial length shortening.

\section{References}

[1] Gong Q, Janowski M, Luo M, et al. Share Efficacy and Adverse Effects of Atropine in Childhood Myopia: A Meta-analysis. JAMA Ophthalmol 2017; 135 (6): 624-30.

[2] Hiraoka T, Kakita T, Okamoto F, et al. Long-term Effect of Overnight Orthokeratology on Axial Length Elongation in Childhood Myopia: A 5-year Follow-up Study. Invest Ophthalmol Vis Sci 2012; 53: 3913-19.

[3] Chamberlain P, Peixoto-de-Matos SC, Logan NS, Ngo C, Jones D, Young G. A 3-year Randomized Clinical Trial of MiSight Lenses for Myopia Control. Optom Vis Sci 2019; 96 (8): 556-67.

[4] A Review of Current Concepts of the Etiology and Treatment of Myopia. Eye Contact Lens 2018; 44 (4): 231- 47.

[5] Nickla DL, et al., Myopic Defocus in the Evening is More Effective at Inhibiting Eye Growth than Defocus in the Morning: Effects on Rhythms in Axial Length and Choroid Thickness in Chicks. Exp Eye Res 2017; 154: 104-15.

[6] Burfield HJ, Patel NB, and Ostrin LA, Ocular Biometric Diurnal Rhythms in Emmetropic and Myopic Adults. Invest Ophthalmol Vis Sci 2018. 59 (12): 5176-87.
[7] Xiong F, Mao T, Liao H, etc. Orthokeratology and Low-Intensity Laser Therapy for Slowing the Progression of Myopia in Children. Biomed Res Int. 2021 Jan 27; 2021: 8915867.

[8] Sankaridurg P, Bakaraju RC, Naduvilath T, Chen X, Weng R, Tilia D, Xu P, Li W, Conrad F, Smith EL 3rd, Ehrmann K. Myopia Control with Novel Central and Peripheral Plus Contact Lenses and Extended Depth of Focus Contact Lenses: 2 Year Results from a Randomised Clinical Trial. Ophthalmic Physiol Opt 2019; 39 (4): 294-307.

[9] Wang M, Schaeffel F, Jiang B, Feldkaemper M. Effects of Light of Different Spectral Composition on Refractive Development and Retinal Dopamine in Chicks. Invest Ophthalmol Vis Sci. 2018 Sep 4; 59 (11): 4413-24.

[10] Foulds WS, Barathi VA, and Luu CD, Progressive Myopia or Hyperopia can be Induced in Chicks and Reversed by Manipulation of the Chromaticity of Ambient Light. Invest Ophthalmol Vis Sci 2013; 54 (13): 8004-12.

[11] The Effects of Light Sources with Different Spectral Structures on Ocular Axial Length in Rainbow Trout (Oncorhynchus mykiss). Exp Eye Res 2016; 151: 212-21.

[12] Jiang L, et al., Interactions of Chromatic and Lens-induced Defocus During Visual Control of Eye Growth in Guinea Pigs (Cavia Porcellus). Vision Res 2014; 94: 24-32.

[13] Smith EL 3rd, Hung LF, Arumugam B, Holden BA, Neitz M, Neitz J. Effects of Long-Wavelength Lighting on Refractive Development in Infant Rhesus Monkeys. Invest Ophthalmol Vis Sci. 2015; 56 (11): 6490-500.

[14] Gawne TJ, Ward AH, Norton TT. Long-wavelength (red) Light Produces Hyperopia in Juvenile and Adolescent Tree Shrews. Vision Res. 2017 Nov; 140: 55-65.

[15] Ward AH, Norton TT, Huisingh CE, Gawne TJ. Share. The Hyperopic Effect of Narrow-band Long-wavelength Light in Tree Shrews Increases Non-linearly with Duration. Vision Res. 2018 May; 146-147: 9-17.

[16] Karu TI, Pyatibrat LV, Kolyakov SF, Afanasyeva NI. Absorption Measurements of a Cell Monolayer Relevant to Phototherapy: Reduction of Cytochrome C Oxidase Under Near IR Radiation. J Photochem Photobiol B 2005; 81: 98-106.

[17] Zhang Y, Song S, Fong CC, et al. cDNA Microarray Analysis of Gene Expression Profiles in Human Fibroblast Cells Irradiated with Red Light. J Invest Dermatol 2003; 120: 849-57.

[18] Bjordal JM, Couppe C, Chow RT, et al. A Systematic Review of Low Level Laser Therapy with Location-Specific Doses for Pain from Chronic Joint Disorders. Aust J Physiother 2003; 49: 107-16.

[19] Peplow PV, Chung TY, Baxter GD. Laser Photobiomodulation of Wound Healing: a Review of Experimental Studies in Mouse and Rat Animal Models. Photomed Laser Surg 2010; 28: 291-325.

[20] Salehpour F, Mahmoudi J, Kamari F, et al. Brain Photobiomodulation Therapy: a Narrative Review. Mol Neurobiol 2018; 55 (8): 6601-36.

[21] Chung H, Dai T, Sharma SK, et al. The Nuts and Bolts of Low-Level Laser (Light) Therapy. Ann Biomed Eng 2012; 40 (2): 516-33. 
[22] Stausholm MB, Naterstad IF, Joensen J, et al. Efficacy of Low-Level Laser Therapy on Pain and Disability in Knee Osteoarthritis: Systematic Review and Meta-Analysis of Randomised Placebo-Controlled Trials. BMJ Open 2019; 9 (10): $\mathrm{e} 031142$.

[23] Yeh SW, Hong CH, Shih MC, et al. Low-Level Laser Therapy for Fibromyalgia: A Systematic Review and Meta-Analysis. Pain Physician 2019; 22 (3): 241-54.
[24] Merry GF, Munk MR, Dotson RS, et al. Photobiomodulation Reduces Drusen Volume and Improves Visual Acuity and Contrast Sensitivity in Dry Age-Related Macular Degeneration. Acta Ophthalmol 2017; 95 (4): e270-7.

[25] Geneva II. Photobiomodulation for the Treatment of Retinal Diseases: a Review. Int J Ophthalmol 2016; 9 (1): 145-52.

[26] Rucker F. Monochromatic and White Light and the Regulation of Eye Growth. Exp Eye Res 2019; 184: 172-82. 